\title{
Practice-Changing Interventions in the Systemic Management of Breast Cancer
}

\author{
Presented by William J. Gradishar, MD
}

\section{ABSTRACT}

Systemic treatment for metastatic breast cancer now incorporates many targeted agents and a plethora of combinations specific to the breast cancer subtype. New to the treatment paradigm are fam-trastuzumab deruxtecan-nxki, and tucatinib for HER2-positive disease; the PI3K inhibitor alpelisib in combination with fulvestrant for estrogen receptor-positive and PIK3CA-mutated tumors; PARP inhibitors for patients with germline BRCA1/2 mutations; and the anti-PD-L1 agent atezolizumab in combination with albumin-bound paclitaxel for triple-negative disease with PD-L1 mutations in tumors. In addition, for estrogen receptor-positive disease, the role of CDK4/6 inhibitors increased substantially during the past year, as overall survival results have emerged. These targeted agents are greatly improving patient outcomes, and thus have all been incorporated into the NCCN Guidelines for Breast Cancer.

Breast cancer mortality is approximately half of what it was in 1980 due to aggressive screening, combination adjuvant therapies, and the emergence of effective targeted agents for the metastatic setting. "We anticipate that as data emerge from some of the current large trials that are incubating, we will see that trend continuing. Long-term prognosis should be even better for women entering clinical trials in 2020," according to William J. Gradishar, MD, Betsy Bramsen Professor of Breast Oncology, and Chief of Hematology/Oncology, Robert H, Lurie Comprehensive Cancer Center of Northwestern University.

At the NCCN 2020 Virtual Annual Conference, Dr. Gradishar informed listeners about practice-changing interventions for metastatic breast cancer, and how these interventions have been incorporated into the NCCN Clinical Practice Guidelines in Oncology (NCCN Guidelines) for Breast Cancer. He noted that even in this era of molecular medicine, clinicians still rely on "very pragmatic decision-making." This decision-making starts with breast cancer subtype: whether the tumor expresses the estrogen receptor (ER) and/or progesterone receptor and/or the HER2 protein, whether it lacks all these characteristics and is therefore triplenegative, and, more recently, "teasing out" the subset of patients with BRCA 1/2-associated disease. Dr. Gradishar organized his updates around these subtypes.

\section{HER2-Positive Disease}

The benefit of HER2-targeted therapies on overall survival (OS) for patients with metastatic HER2-positive breast cancer has been revolutionary, especially after the introduction of dual-targeted therapy, and this has continued to be refined in numerous trials. Dual targeting with trastuzumab and pertuzumab plus a taxane is now the preferred first-line option for metastatic disease based on the robust results of the pivotal CLEOPATRA trial. ${ }^{1}$

On disease progression, T-DM1 is the preferred treatment option. Thereafter, a number of combinations can be utilized, including capecitabine/lapatinib, trastuzumab/lapatinib, trastuzumab/chemotherapy, pertuzumab (if not yet given) or endocrine therapy (as indicated) + anti-HER2 agent. Clinicians can expect several novel agents to be added to the guidelines soon, he said. "We try to leverage these tools, switching to a different HER2-directed therapy, as disease progresses. As long as the patient appears to be benefitting, and there are reasonable expectations, we continue systemic therapy, with shared decision-making," he commented.

A recent addition to the guidelines, fam-trastuzumab deruxtecan-nxki (T-Dxd), was added based on an impressive presentation at the 2019 San Antonio Breast Cancer Symposium, he said. ${ }^{2}$ As an antibody-drug conjugate, T-Dxd is similar to T-DM1, however, because the payload is a topoisomerase- 1 inhibitor, there is a higher drug-to-antibody ratio, potentially greater antitumor effect, and potentially a bystander effect that may produce broader killing, he noted. The phase II DESTINY-Breast01 trial enrolled patients who had undergone a median of 6 prior lines of therapy, $13 \%$ of whom had stable brain metastases. ${ }^{2}$ The response rate to 
T-Dxd in this challenging population was $60.9 \%$, disease control rate was $97.3 \%$, and median progression-free survival (PFS) was 16 months. ${ }^{3}$

"These results are what excited us in San Antonio, and what led to the FDA-approval of T-Dxd on December 20, 2019," Dr. Gradishar commented. Most adverse events with the drug are mild, but interstitial lung disease, including fatal cases, has been observed, which prompted a black box warning. Clinicians need to be vigilant for this toxicity and have a low threshold for stopping or dose-reducing T-Dxd, he cautioned.

Also presented in San Antonio were updated data on the oral HER2-selective tyrosine kinase inhibitor tucatinib. In the HER2CLIMB trial examining 612 heavily pretreated patients, tucatinib + trastuzumab/capecitabine versus trastuzumab/capecitabine doubled response rates and significantly improved median PFS (7.8 vs 5.6 months; hazard ratio $[\mathrm{HR}], 0.54 ; P<.0001)$ and OS (21.9 vs 17.4 months; HR, $0.66 ; P<.00480) .{ }^{4}$ "This was pretty remarkable," Dr. Gradishar exclaimed. "Based on these findings, tucatinib was recently FDA-approved as well." [Editor's note: tucatinib was added to the NCCN Guidelines in May 2020.]

"Many drugs are currently being evaluated posttrastuzumab and T-DM1-not only new agents, but also combinations with other targeted therapies," he added (Figure 1). One of these interesting novel compounds is margetuximab, which is Fc-engineered in a manner that increases its affinity for the activating Fc receptor genotype and decreases its affinity for the inhibitory genotype. Investigators for the phase III SOPHIA trial presented updated results at the 2019 San Antonio Breast Cancer Symposium, which evaluated margetuximab + chemotherapy versus trastuzumab/chemotherapy in the third-line or greater. ${ }^{5}$ Although the overall population did not benefit, within the subset of patients who were CD16A-185 carriers (FF or $\mathrm{FV}$ ), those treated with margetuximab

\begin{tabular}{|c|c|c|}
\hline Novel Anti-HER2 & Antibody-Drug & \\
\hline Antibodies & Conjugates & \\
\hline $\begin{array}{l}\text { - Margetuximab } \\
\text { - ZW25 }\end{array}$ & $\begin{array}{l}\text { - DS 8201a } \\
\text { - SYD985 }\end{array}$ & Promising combinatory \\
\hline - MCLA-128 & - ARX788 & agents \\
\hline - GBR 1302 & - Medi4276 & - Immune therapies/IO \\
\hline - PRS-343 & - XMT-1522 & - $\operatorname{CDK} 4 / 6$ \\
\hline - BTRC4017a & - ADCT 502 & - PI3K inhibitor \\
\hline - MM-III & - Pf-06804103 & - Other \\
\hline Small Molecule & $\begin{array}{l}\text { - A166 } \\
\text { - DHES0815A }\end{array}$ & \\
\hline $\begin{array}{l}\text { Inhibitors } \\
\text { - Pyrotinib } \\
\text { - Poziotinib } \\
\text { - TAS-0728 }\end{array}$ & $\begin{array}{l}\text { - ALT-P7 } \\
\text { - ZW49 } \\
\text { - RC48 }\end{array}$ & \\
\hline
\end{tabular}

Figure 1. Emerging therapies for HER2-positive breast cancer. survived 4.3 months longer (HR, $0.79 ; P=.087$ ). Based on these results, margetuximab is now under consideration by the FDA.

Novel drugs such as the new HER2-directed agents do come at a high price, Dr. Gradishar acknowledged. To that end, several biosimilars are now on the market: trastuzumab-dkst, trastuzumab-anns, trastuzumab-dttb, trastuzumab-pkrb, and trastuzumab-gyyp. "These are reflected in the NCCN Guidelines as appropriate choices to consider when you might be using trastuzumab," he said.

\section{ER-Positive Breast Cancer}

For ER-positive breast cancer, a number of targeted therapies are now combined with endocrine therapy. These therapies include everolimus, inhibitors of cyclin-D kinases 4 and 6 (CDK4/6), and, most recently a PI3K inhibitor, alpelisib (Figure 2). Treatment combinations have emerged based on an understanding of the biology of this subtype: that many signaling pathways exhibit cross talk and become dominant when cells become resistant to antiestrogen therapy. With knowledge of these pathways, these targets became druggable, he said.

First-line preferred regimens include endocrine therapy plus 1 of the 3 CDK4/6 inhibitors: abemaciclib, palbociclib, or ribociclib (all category 1). In subsequent lines, preferred regimens allow for endocrine therapy plus the inclusion of a CDK4/6 inhibitor if not previously used, and alpelisib in combination with fulvestrant for PIK3CA-mutated tumors. Abemaciclib can be useful as monotherapy in some circumstances.

CDK4/6 inhibitors have shown robust ability to improve outcomes in the first- and second-line metastatic settings, consistently showing a doubling in PFS and maintaining efficacy in postmenopausal women as well as premenopausal women rendered postmenopausal by ovarian suppression.

Recently, an OS advantage was also reported for ribociclib/fulvestrant in MONALEESA-3 study in the firstline setting (HR, 0.724; $P=.00455)^{6}$ and for abemaciclib/ fulvestrant in the MONARCH 2 trial in the early relapse/ second-line setting (HR, 0.730; 95\% CI, 0.530-1.004). ${ }^{7}$ "Regardless of the setting, the addition of a CDK4/6 inhibitor made a significant improvement in outcome and it's clearly a class effect," he said. Without a clear understanding of which patients benefit most from these drugs, all women with ER-positive/HER2-negative tumors should be considered for endocrine therapy plus a CDK4/6 inhibitor, he said.

As for the small subset of patients with PIK3CAmutated tumors, results have shown that alpelisib + fulvestrant significantly improved median PFS, from 5.7 months with placebo to 11.0 months (HR, 0.65; $P=.00065) .{ }^{8}$ Alpelisib is most beneficial when used after disease progression on a CDK4/6 inhibitor. ${ }^{9}$ 


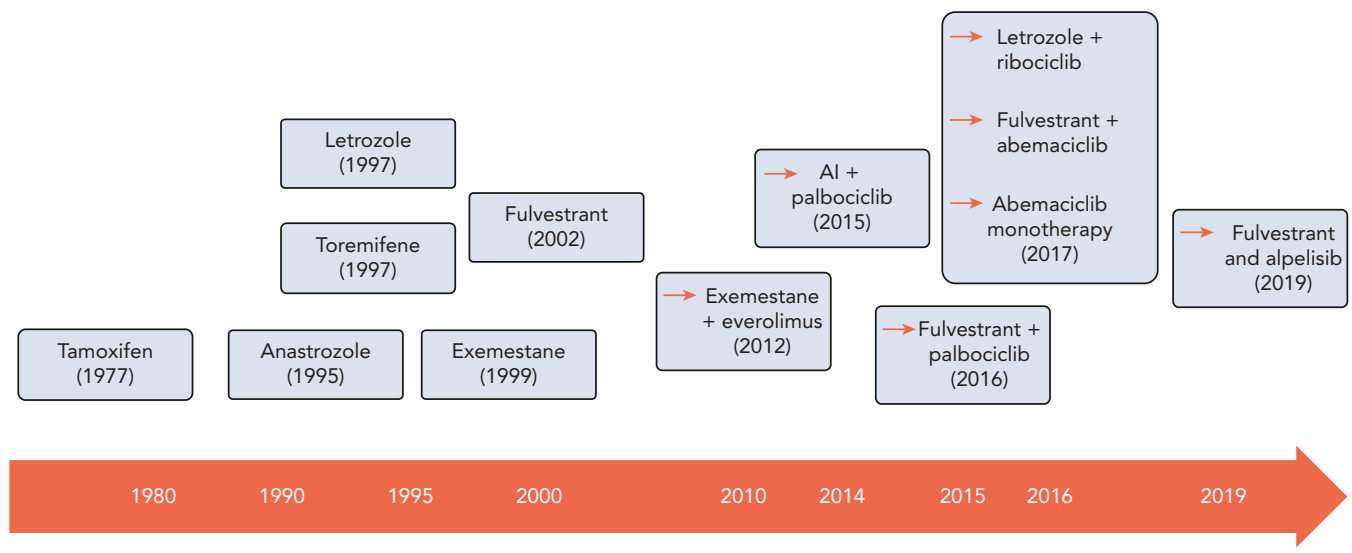

Figure 2. Examples of hormonal therapies for ER+ breast cancer: evidence of recent acceleration.

Abbreviations: Al, aromatase inhibitor; ER, estrogen receptor.

Selective estrogen receptor downregulators/degraders (SERDs) inhibit the dimerization of the ER and are being explored for their use in advanced, progressive ER-positive, metastatic breast cancer, including those in ER mutations (ESR1). Several next-generation SERDs are in early-phase trials, which could soon join a growing list of novel approaches for treating ER-positive breast cancer after CDK4/6 inhibition.

"The hope is we'll have more agents to utilize in this large group of patients," he said. "This is an attractive target that we want to exploit."

\section{Triple-Negative and BRCA-Mutated Breast Cancer}

"Triple-negative breast cancer strikes fear in patients," Dr. Gradishar commented. This subtype is intrinsically complex, marked by many somatic mutations and rearrangements. "To think that any one therapy will affect their growth is probably overly simplistic," he said. The choice of systemic treatment now takes into account certain characteristics that can be exploited, in particular, germline $B R C A 1 / 2$ mutations, which can be targeted by inhibitors of PARP and expression of PD-L1, a biomarker for potential benefit from immunotherapy.

Olaparib and talazoparib have both been shown to improve PFS in patients with germline BRCA mutations, ${ }^{10,11}$ and are considered options for these patients. However, the benefit of these drugs is optimized when they are given earlier in the course of metastatic disease rather than later, he added.

Less frequently, microsatellite instability-high (MSI-H) status can also be exploited with the anti-PD-L1 agent atezolizumab + nab-paclitaxel. The rate NTRK fusions can be targeted with larotrectinib and entrectinib (Figure 3).

Although PD-1/PD-L1 inhibitors have a solid place in many other malignancies, the data are limited as to their benefit in breast cancer. The Impassion 130 study

ADDITIONAL TARGETED THERAPIES AND ASSOCIATED BIOMARKER TESTING FOR RECURRENT OR STAGE IV (M1) DISEASE

\begin{tabular}{|c|c|c|c|c|c|}
\hline \multicolumn{6}{|c|}{ Biomarkers Associated with FDA-Approved Therapies } \\
\hline $\begin{array}{l}\text { Breast Cancer } \\
\text { Subtype }\end{array}$ & Biomarker & Detection & $\begin{array}{l}\text { FDA-Approved } \\
\text { Agents }\end{array}$ & $\begin{array}{l}\text { NCCN Category } \\
\text { of Evidence }\end{array}$ & $\begin{array}{l}\text { NCCN Category } \\
\text { of Preference }\end{array}$ \\
\hline \multirow[t]{2}{*}{ Any $^{\mathrm{a}}$} & \multirow{2}{*}{$\begin{array}{l}\text { BRCA1 mutation } \\
\text { BRCA2 mutation }\end{array}$} & \multirow[t]{2}{*}{ Germline sequencing } & Olaparib & Category 1 & Preferred \\
\hline & & & Talazoparib & Category 1 & Preferred \\
\hline $\begin{array}{l}\text { HR-positive/ } \\
\text { HER2-negative }^{b}\end{array}$ & PIK3CA mutation & $\begin{array}{l}\text { PCR (blood or tissue block if blood negative), } \\
\text { molecular panel testing }\end{array}$ & $\begin{array}{l}\text { Alpelisib }+ \\
\text { fulvestrant }^{d}\end{array}$ & Category 1 & $\begin{array}{l}\text { Preferred second- } \\
\text { line therapy }\end{array}$ \\
\hline $\begin{array}{l}\text { HR-positive/ } \\
\text { HER2-negative }\end{array}$ & $\begin{array}{l}\text { PD-L1 expression } \\
\text { - Threshold for positivity: } \\
\geq 1 \% \text { on tumor- } \\
\text { infiltrating immune cells }\end{array}$ & $\mathrm{IHC}$ & $\begin{array}{l}\text { Atezolizumab + } \\
\text { albumin-bound } \\
\text { paclitaxel }\end{array}$ & Category $2 \mathrm{~A}$ & Preferred \\
\hline \multirow[t]{2}{*}{ Any } & \multirow[t]{2}{*}{ NTRK fusion } & \multirow[t]{2}{*}{ FISH, NGS, PCR (tissue block) } & Larotrectinib $^{e}$ & Category 2A & \multirow{2}{*}{$\begin{array}{l}\text { Useful in certain } \\
\text { circumstances }^{e} \\
\text { Useful in certain } \\
\text { circumstances }^{\mathrm{e}}\end{array}$} \\
\hline & & & Entrectinib $^{e}$ & Category $2 \mathrm{~A}$ & \\
\hline Any & MSI-H/dMMR & IHC, PCR (tissue block) & Pembrolizumab $^{f}$ & Category $2 \mathrm{~A}$ & $\begin{array}{l}\text { Useful in certain } \\
\text { circumstances }^{f}\end{array}$ \\
\hline
\end{tabular}

Figure 3. NCCN Guidelines for Invasive Breast Cancer: additional targeted therapies and associated biomarker testing for recurrent or stage IV (M1) disease. Version 4.2020. CNCCN. All rights reserved. 
showed benefit for atezolizumab + nab-paclitaxel in previously untreated patients who expressed PD-L1. ${ }^{12}$ Based on a 38\% reduction in disease progression $(P<.001)$ and a $29 \%$ reduction in mortality, atezolizumab was FDA-approved and is listed in the NCCN Guidelines.

\section{Curing More Women}

In closing, Dr. Gradishar reminded listeners, "We already cure metastatic breast cancer" with adjuvant therapy for micrometastatic disease, various approaches for oligometastatic lesions and locoregional recurrences, and intelligent use of chemotherapy. "The question is, what can we do to improve the cure rate?" he said. This will require finding and treating smaller metastases (and eliminating disparities that lead to delayed diagnosis and treatment), developing the right drugs for the right patients (teasing out the subtypes), understanding the effect of prior therapies and the best options for current treatment, and using an increasing array of molecular markers to make decisions, he said.

"Biology is driving drug development," he said. "The hope is that in early- and late-stage disease, outcomes will be improved."

Disclosures: Dr. Gradishar has disclosed that he is a scientific advisor for AstraZeneca Pharmaceuticals LP; MacroGenics, Inc.; Roche Laboratories, Inc./Genentech, Inc.; and Seattle Genetics, Inc.

Correspondence: William J. Gradishar, MD, Department of Hematology/Oncology, Robert H. Lurie Comprehensive Cancer Center of Northwestern University, 676 North St. Clair Street, Suite 850, Chicago, IL 60611. Email: w-gradishar@northwestern.edu

\section{References}

1. Swain SM, Baselga J, Kim SB, et al. Pertuzumab, trastuzumab, and docetaxel in HER2-positive metastatic breast cancer. N Engl J Med 2015; 372:724-734.

2. Krop IE, Saura C, Yamashita T, et al. [Fam-] trastuzumab deruxtecan (T-XDd;DS-8021a) in subjects with HER2-positive metastatic breast cancer previously treated with T-DM1: a phase 2, multicenter, open-label study (DESTINY-Breast01) [abstract]. Presented at the 2019 San Antonio Breast Cancer Symposium; December 8-12, 2020; San Antonio, Texas. Abstract GS1-03.

3. Modi S, Saura C, Yamashita T, et al. Trastuzumab deruxtecan in previously treated HER2-positive breast cancer. N Engl J Med 2020;382:610-621.

4. Murthy RK, Loi S, Okines A, et al. Tucatinib vs placebo added to trastuzumab and capecitabine for patients with pretreated HER2+ metastatic breast cancer with and without brain metastases (HER2CLIMB) [abstract] Presented at the 2019 San Antonio Breast Cancer Symposium; December 8-12, 2020; San Antonio, Texas. Abstract GS1-01.

5. Rugo HS, Im S, Cardoso F, et al. Phase 3 SOPHIA study of margetuximab + chemotherapy vs trastuzumab + chemotherapy in patients with HER2 + metastatic breast cancer after prior anti-HER2 therapies: second interim overall survival analysis [abstract]. Presented at the 2019 San Antonio Breast Cancer Symposium; December 8-12, 2020; San Antonio, Texas. Abstract GS1-02.

6. Slamon DJ, Neven P, Chia $\mathrm{S}$, et al. Overall survival results of the phase III MONALEESA-3 trial of postmenopausal patients with hormone receptorpositive, human epidermal growth factor 2-negative advanced breast cancer treated with fulvestrant + ribociclib [abstract]. Presented at the ESMO Congress 2019; September 27-October 1, 2019; Barcelona, Spain Abstract LBA7.

7. Sledge GW Jr, Toi M, Neven P, et al. The effect of abemaciclib plus fulvestrant on overall survival in hormone receptor-positive, ERBB2-negative breast cancer that progressed on endocrine therapy-MONARCH 2: a randomized clinical trial. JAMA Oncol 2019;6:116-124.

8. Andre F, Ciruelos E, Rubovszky G, et al. Alpelisib for PIK3CA-mutated, hormone receptor-positive advanced breast cancer. N Engl J Med 2019; 380:1929-1940.

9. Juric D, Ciruelos EM, Rubovszky G, et al. Alpelisib (ALP) + fulvestrant (FUL) for advanced breast cancer (ABC): phase 3 SOLAR-1 trial results [abstract]. Presented at the 2018 San Antonio Breast Cancer Symposium; December 4-8, 2018; San Antonio, Texas. Abstract GS3-08.

10. Robson M, Im SA, Senkus E, et al. Olaparib for metastatic breast cancer in patients with a germline BRCA mutation. N Engl J Med 2017;377: 523-533.

11. Litton JK, Rugo HS, Ettl J, et al. Talazoparib in patients with advanced breast cancer and a germline BRCA mutation. N Engl J Med 2018;379. 753-763.

12. Schmid P, Adams S, Rugo HS, et al. Atezolizumab and nab-paclitaxel in advanced triple-negative breast cancer. N Engl J Med 2018;379: 2108-2121. 